EPJ Web of Conferences 87,01001 (2015)

DOI: $10.1051 /$ epjconf/ 20158701001

(C) Owned by the authors, published by EDP Sciences, 2015

\title{
Summary for the Theory Session at EC-18
}

\author{
N.B. Marushchenko ${ }^{1}$ \\ ${ }^{1}$ Max Planck Institute for Plasma Physics, EURATOM Association, Wendelsteinstr. 1, D-17491 Greifswald, Germany
}

\begin{abstract}
A brief review of the theory contributions presented in the EC-18 Workshop is given. The covered spectrum of topics is quite broad including the electron cyclotron wave propagation physics as well the results of numerical modelling of transport and current drive.
\end{abstract}

\section{Introduction}

From a total of thirteen theoretical contributions (eight orals and five posters), presented in EC-18 Workshop, eleven reports were presented in the Theory session and two reports were formally included in the ECE session. The topics covered in the presentations are the following: propagation of EC waves in plasmas with density fluctuations (3 papers), Spitzer problem and current drive (4 papers), radiative transport and ECE diagnostic (3 papers), ITER design ( 2 paper) and W7-X scenarios (1 paper).

\section{Propagation of EC waves in plasmas with density fluctuations}

O. Maj gave a talk where the scattering of RF beams in the electron cyclotron frequency range by turbulent density fluctuations in tokamak plasmas was considered. In this work, the Wigner transform and Weyl symbol calculus were applied to the relevant integro-differential equation for the wave electric field, thus obtaining a kinetic radiative transfer model which is general enough to account for both diffraction and scattering effects. For numerically solving the wave kinetic equation, the recently developed code WKBeam [1] was applied. First results show that, for ITER, the beam might suffer from significant diffusion due to fluctuations, i.e. significant broadening of the deposition profile. Contrary to that, scattering effects were found to be negligible in medium-size tokamaks such as ASDEX Upgrade.

In the talk of A. Ram, the scattering of a plane EC wave on single density blobs was investigated with full wave modeling. The plasma, both inside and outside the blobs, is assumed to be homogeneous with arbitrary densities in either region; thus, the model is not limited to small density fluctuations, i.e. $\delta n_{e} / n_{e}$ is arbitrary. Numerically, the scattering of both O- and X-modes and the coupling of them due to the scattering were studied for different $\delta n_{e} / n_{e}$ and blob-sizes, $a$, from $a / \lambda \gg 1$ to $a / \lambda \sim 1$ (here, $\lambda$ is the wave-length).
A. Köhn gave a talk devoted to the influence of density fluctuations on $\mathrm{O}-\mathrm{X}$ mode conversion and microwave propagation. Using 2D full-wave simulations, it was shown that a perturbation having a size on the order of the vacuum wavelength $\lambda$ of the injected microwave has the largest influence. In particular, the effect of a single blob is significant if (a) the blob is within beam width, (b) the blob is wider than $\lambda / 3$, and (c) the blob density is related to the cut-off density as $\delta n_{e}>n_{e, \text { cut }} / 5$. It was also found that the scattering of microwaves is the largest if the average structure size of turbulent fluctuations is of the order of the wave-length, i.e. $\langle a\rangle / \lambda \sim 1$.

\section{Current drive and Spitzer problem}

In the talk of E. Westerhof, a new closure relation for the EC driven current density appearing in the modified Ohm's law $\mathbf{E}+\mathbf{v} \times \mathbf{B}=\eta\left(\mathbf{J}-\mathbf{J}_{\mathrm{ECCD}}\right)$ for single fluid MHD modeling was presented. The phenomenological model is based on representation of the EC current drive as combination of two contributions, $J_{\mathrm{ECCD}} \equiv J^{-}+J^{+}$, coming from asymmetric collisional relaxation of the "hole" and "bulge" in velocity space, respectively, driven by quasilinear diffusion in perpendicular direction. The new relation faithfully represents the nonlocal character of the EC driven current and its main origin in the Fisch-Boozer effect and contains the closure relation of Giruzzi et al. [2] as one of its limits. The closure relation is validated by both an analytical solution of an approximated Fokker-Planck equation as well as by full bounce-averaged, quasi-linear Fokker-Planck code simulations of ECCD inside rotating magnetic islands.

In the paper of S. Murakami progress in development of momentum conserving Monte-Carlo code suitable for ECCD study in stellarators was presented. Numerical simulations of ECCD generation in helical plasmas was performed by the GNET code [3] which solves a linearized drift kinetic equation in 5D phase space. Conservation of toroidal momentum in the collision operator has been implemented in the GNET code using an iteration scheme. In 
all studied cases, good convergence after the 4th iteration has been obtained.

W. Kernbichler gave a talk about the effect of finite collisionality on the global ECCD efficiency in tokamaks. Simulations have been performed with help of the generalized Spitzer function precalculated by the drift kinetic equation solver NEO-2 [4], which was applied as input for the ray-tracing code TRAVIS [5]. It was shown that in contrast to the asymptotic low and high collisionality limits, where the symmetric part of the Spitzer function is small, the direction of the current may become independent of the microwave beam launch angle in ECCD scenarii with relatively low single-pass absorption such as $\mathrm{O} 2$ and $\mathrm{X} 3$. This effect appears in the studied scenarii since the symmetric part of the Spitzer function localized around the trapped particle region due to finite collisionality becomes comparable to its antisymmetric part.

Y. R. Lin-Liu presented an analytical model for estimation of the upper bound of the effects of synchrotron radiation losses on the Ohmic conductivity, $\Delta \sigma / \sigma$, in uniform magnetized relativistic plasmas. The radiation losses are treated as a perturbation to Coulomb collisions modeled by relativistic collision operator. For the frictionforce due to synchrotron radiation the model which was heuristically derived in Ref. [6] has been applied. Diffusion of electrons in velocity space due to emission and absorption of synchrotron radiation is ignored. Corrections to the Ohmic conductivity are expressed as a function of electron plasma pressure, temperature, and the effective ion charge, $\Delta \sigma / \sigma=f\left(\beta_{e}, T_{e} / m_{e} c^{2}, Z_{\mathrm{eff}}\right)$. It was found that for the DEMO parameters with $T_{e} \simeq 50 \mathrm{keV}$, the reduction of conductivity does not exceed $5 \%$.

\section{Radiative transport and ECE diagnostic}

F. Albajar presented results of a comparative study of EC wave and bremsstrahlung contributions to the local and total power balance in relation to the $\alpha$-particle heating power. Simulations were performed using the RAYTEC code [7] for a range of plasma conditions, wall reflection coefficients of the EC waves, and magnetic fields relevant to DEMO. The RAYTEC parametric results corroborate that EC wave losses, even for a wall reflection coefficient as high as 0.8 (realistic values are expected to be lower), are significant for a peak electron temperature of $40 \mathrm{keV}$ and EC wave losses become an essential loss channel for still higher temperatures. In all cases EC power losses are appreciably larger than bremsstrahlung effects (the higher the electron temperature is).

In presentation of P. V. Minashin, a model of multipass absorption of external EC radiation for the initial stage of tokamak discharge was suggested. The main assumptions adopted in the model are the following: (a) the EC radiation injected in the chamber becomes isotropic and uniform after the first reflection from the wall; (b) due to polarization scrambling in wall reflections both $\mathrm{O}$ and $\mathrm{X}$-modes exist, but only the $\mathrm{X}$-mode is completely absorbed during a single pass. The model is successfully implemented in the CYNEQ code [8] and applied for studying the initial stage of EC discharges in ITER.
The next presentation of P. V. Minashin was devoted to ECE diagnostics in hot plasmas with DEMO-like parameters. Using the code CYNEQ, the contribution from the supra-thermal electrons in the radiative spectra was studied. The anisotropic bi-Maxwellian electron distribution function was applied with $T_{e}=30 \mathrm{keV}$ in the bulk and $10 \%$ hot electrons with $T_{\perp}^{\text {hot }}=2 T_{e}$. Analyzing the radiative spectrum with all significant contributions from the cyclotron harmonics (in these calculations, $n \leq 14$ ), the authors suggest that the energy spectrum of suprathermal electrons in range of $E_{\perp} \sim(2-3) T_{\perp}^{\text {hot }}$ can be restored.

In the talk of M. van Berkel, the problem of data extraction from a noisy ECE signal is considered. It was shown that studying the stochastic properties of ECE noise one can arrive at better estimates of some indirectly measured quantities. As examples, real-time detection of the sawtooth instability period and estimation of the thermal diffusivity based on ECE-measurements were discussed.

\section{ITER and W7X design}

E. Poli analyzed in his talk the criteria guiding the design of the upper electron-cyclotron launcher (UL) for ITER. The stabilization criteria was re-derived to assess their validity limit and to analyze the relative importance of different beam-broadening effects. In particular, in order to find an upper limit to the expected transport level, the diffusion of fast electrons was investigated by means of gyrokinetic simulations in the absence of islands. It was found that, according to beam-tracing and stabilization criteria, the installed EC power for UL is well within the margin for complete NTM suppression in ITER. It was also shown that the beam scattering due to density fluctuations is likely to be most deleterious for localized CD profile (to be further investigated).

A presentation of L. Figini was also devoted to the UL for EC waves in ITER. The performance of the UL was assessed and requirements on its design to achieve all the required functionalities were established. Different scenarios were studied, along the full temporal evolution of the discharge, including the reference ITER 15 MA H-mode plasma with $B=5.3 \mathrm{~T}$, a case with reduced magnetic field strength at $2.65 \mathrm{~T}$, and a steady state scenario. The steering range necessary to reach the $q=3 / 2$ and $q=2$ surfaces was determined for the different scenarios. The optimal launching angles and the power required for NTMs stabilization was estimated and found to be sufficient in the main phases of the discharge. The increase in power requirements due to partial misalignment among the beams launched from the same mirror was also estimated.

N. B. Marushchenko presented a talk about selective ECR heating of trapped/passing electrons by X3-mode near the minimum of $B$ in the W7X stellarator. The main power for X2-mode heating is expected to be launched from a low-field side (LFS) port near the maximum of $B$, while for the X3-mode high-field side (HFS) launch from the "remote-steering launcher" (RSL) [10] is foreseen, which is located near the plane where $B$ has a minimum and $\nabla B$ is weak. For two magnetic equilibriums with 
the different "mirror-ratio", $B_{\max } / B_{\min }=1.36$ and 1.64, two different scenarii were considered. Using the transport code [9] and ray-tracing code TRAVIS [5] coupled together, it was shown that by change of the launch direction by RSL or switching (on/off) of gyrotrons, the fraction of power absorbed from the X3 beam can be redirected from passing electrons to locally trapped ones. In experiments with rapid modulation of $P_{\text {abs }}^{\text {trap }} / P_{\text {abs }}^{\text {total }}$, additional convective (non-neoclassical) contributions to the radial transport can be expected.

\section{References}

[1] H. Weber, IPP report 5/134 (2013).
[2] G. Giruzzi et al., Nucl. Fusion 39, 107 (1999).

[3] S. Murakami et al., Nucl. Fusion 40, 693 (2000).

[4] W. Kernbichler et al., J. Plasma and Fusion Res. 3, 51061 (2008).

[5] N. B. Marushchenko, Y. Turkin and H. Maassberg, Comp. Phys. Commun. 185, 165 (2014).

[6] I. B. Bernstein and D. C. Baxter, Phys. Fluids 24, 108 (1981).

[7] F. Albajar, M. Bornatici and F. Engelmann, Nucl. Fusion 49, 115017 (2009)

[8] A. B. Kukushkin and P. V. Minashin, Proc. 24th IAEA Fusion Energy Conf. TH/P6-25 (2012)

[9] Y. Turkin et al., Fusion Sci. Technol. 50, 387 (2006)

[10] W. Kasparek et al., this conference. 